\title{
Inorganic Chemistry
}

\section{Supporting Information}

\section{Smart grafting of lanthanides onto silica via $\mathrm{N}, \mathrm{N}$-dialkylcarbamato complexes}

Lidia Armelao, ${ }^{\dagger}$ Daniela Belli Dell’Amico, ${ }^{\ddagger}$ Luca Bellucci, ${ }^{\ddagger}$ Gregorio Bottaro, ${ }^{\dagger}$ Luca Labella, ${ }^{*}$ Fabio Marchetti ${ }^{\star}$ and Simona Samaritani*

${ }^{\star}$ Dipartimento di Chimica e Chimica Industriale, Università di Pisa, via Giuseppe Moruzzi 13, I-56124

${ }^{\dagger}$ CNR IENI and INSTM, Dipartimento di Scienze Chimiche, Università di Padova, via Marzolo 1, I-35131Padova

S1

Synthesis of $\left[\mathbf{T m}\left(\mathrm{O}_{2} \mathbf{C N B u}\right)_{3}\right]$. A solution of dibutylamine $(12.6 \mathrm{~mL}, 74.8 \mathrm{mmol})$ in heptane (50 $\mathrm{mL}$ ) was saturated with carbon dioxide and then added to $22.4 \mathrm{~mL}$ of an aqueous solution of thulium chloride $(6.22 \mathrm{mmol})$ at $0{ }^{\circ} \mathrm{C}$. After shaking for a few seconds at $0{ }^{\circ} \mathrm{C}$, the organic layer was separated and evaporated at reduced pressure $\left(1.0 \times 10^{-3}\right.$ Torr $)$. A colorless oil was obtained which was dissolved in a few $\mathrm{mL}$ of heptane. The solution was evaporated again and the process was repeated twice. A colorless solid corresponding to $\left[\mathrm{Tm}\left(\mathrm{O}_{2} \mathrm{CNBu}_{2}\right)_{3}\right]$ was obtained $(3.37 \mathrm{~g} ; 4.92$ mmol, $79 \%$ yield). Anal. Calcd for $\mathrm{C}_{27} \mathrm{H}_{54} \mathrm{~N}_{3} \mathrm{TmO}_{6}: \mathrm{CO}_{2}$, 19.3; Tm, 24.6. Found: $\mathrm{CO}_{2}$, 19.8; Tm: $24.0 \%$. ATR-FTIR: $\left(1700-1300 \mathrm{~cm}^{-1}\right): 1588 \mathrm{~s}, 1489 \mathrm{~s}, 1424 \mathrm{~s}, 1375 \mathrm{~m}, 1314 \mathrm{~m}$.

S2

Reaction of $\left[\mathrm{Tb}\left(\mathrm{O}_{2} \mathrm{CNBu}\right)_{3}\right]$, with $\mathrm{Hdbm}$ and characterization of $\left[\mathrm{NH}_{2} \mathrm{Bu} \mathrm{u}_{2}\right]\left[\mathrm{Tb}(\mathrm{dbm})_{4}\right]$, $\left[\mathbf{T b d b m}_{3}\right]$, and $\left[\mathbf{T b}(\mathbf{d b m})_{3}(\mathbf{D M E})\right]$. A colorless solution of $\left[\mathrm{Tb}\left(\mathrm{O}_{2} \mathrm{CNBu}_{2}\right)_{3}\right](1.35 \mathrm{~g} 2.00 \mathrm{mmol})$ in dimethoxyethane (DME, $50 \mathrm{~mL}$ ) was treated with 1,3-diphenyl-1,3-propandione (dibenzoylmethane, Hdbm) (2.02 g; $9.01 \mathrm{mmol})$. A light yellow precipitate progressively formed with carbon dioxide evolution. After $4 \mathrm{~h}$ stirring the suspension was slowly evaporated to dryness $(2 \mathrm{~h})$ at $35^{\circ} \mathrm{C}$. The light yellow residue was suspended in DME, filtrated, washed with DME $(2 \times$ $15 \mathrm{~mL}$ ) and dried in vacuo at RT (4 h). A light yellow solid was recovered $2.13 \mathrm{~g}$ (89.8 \% yield). Anal. Calcd for $\mathrm{TbC}_{68} \mathrm{H}_{64} \mathrm{O}_{8} \mathrm{~N}$ : C, 69.1; H, 5.5; N, 1.2; Tb 13.4 Found: C, 68.7; H, 5.5; N, 1.2; Tb $13.5 \%$. ATR-FTIR $\left(3100-2800 \mathrm{~cm}^{-1}\right): 3058 \mathrm{w}, 2957 \mathrm{w}, 2929 \mathrm{w}, 2871 \mathrm{w} ;\left(1600-900 \mathrm{~cm}^{-1}\right): 1595 \mathrm{~s}$, $1551 \mathrm{~s}, 1508 \mathrm{~s}, 1470 \mathrm{~s}, 1455 \mathrm{~s}, 1422 \mathrm{vs}, 1307 \mathrm{~m}, 1280 \mathrm{~m}, 1217 \mathrm{~m}, 1179 \mathrm{w}, 1154 \mathrm{w}, 1066 \mathrm{~m}, 1022 \mathrm{~m}$, 940w.

Thermal treatment of the solid $\left[\mathrm{NH}_{2} \mathrm{Bu}_{2}\right]\left[\mathrm{Tb}(\mathrm{dbm})_{4}\right](1.59 \mathrm{~g} ; 1.34 \mathrm{mmol})$ at $150{ }^{\circ} \mathrm{C}$ in vacuo for $6 \mathrm{~h}$ yielded the derivative $\left[\mathrm{Tb}(\mathrm{dbm})_{3}\right]$, (weight loss $0.49 \mathrm{~g} v s$ theoretical weight loss $0.48 \mathrm{~g}$ ). Anal. Calcd for $\mathrm{C}_{45} \mathrm{H}_{33} \mathrm{O}_{6} \mathrm{~Tb}$ : Tb 19.2 Found: Tb $18.9 \%$. ATR-FTIR (3100-2800 $\left.\mathrm{cm}^{-1}\right): 3058 \mathrm{~d}, 3026 \mathrm{~d}$; 
$\left(1600-900 \mathrm{~cm}^{-1}\right): 1592 \mathrm{~s}, 1543 \mathrm{~s}, 1508 \mathrm{~s}, 1476 \mathrm{~s}, 1455 \mathrm{~s}, 1422 \mathrm{vs}, 1307 \mathrm{~m}, 1280 \mathrm{~m}, 1217 \mathrm{~m}, 1179 \mathrm{w}$, $1154 \mathrm{w}, 1066 \mathrm{~m}, 1022 \mathrm{~m}, 940 \mathrm{w}$. A portion of $\left[\mathrm{Tb}(\mathrm{dbm})_{3}\right](0.59 \mathrm{~g} ; 0.71 \mathrm{mmol})$ was dissolved in DME $(50 \mathrm{~mL})$ yielding the adduct $\left[\mathrm{Tb}(\mathrm{dbm})_{3}(\mathrm{DME})\right]$. Crystals suitable for single crystal X-Ray diffraction studies were obtained by cooling the solution at $-30{ }^{\circ} \mathrm{C}$. (0.46 g, $70.5 \%$ yield). Anal. Calcd for $\mathrm{C}_{49} \mathrm{H}_{43} \mathrm{O}_{8} \mathrm{~Tb}$ : Tb 17.3 Found: Tb $17.8 \%$. ATR-FTIR $\left(3100-2800 \mathrm{~cm}^{-1}\right): 3060 \mathrm{w}, 3027 \mathrm{w}$, 2940w,2889w, 2832w, (1600-900 cm $\left.\mathrm{cm}^{-1}\right): 1594 \mathrm{~s}, 1549 \mathrm{vs}, 1518 \mathrm{vs}, 1478 \mathrm{~s}, 1459 \mathrm{vs}, 1410 \mathrm{vs}, 1310 \mathrm{~m}$, $1285 \mathrm{~m}, 1220 \mathrm{~m}, 1182 \mathrm{~m}, 1157 \mathrm{w}, 1110 \mathrm{~m}, 1067 \mathrm{~s}, 1023 \mathrm{~m}, 941 \mathrm{~m}$.

\section{S3}

Single-Crystal X-ray Structure Determination of $\left[\mathrm{Tb}(\mathbf{d b m})_{3}(\mathrm{DME})\right]$. The X-ray diffraction experiment was carried out at room temperature $(T=293 \mathrm{~K})$ by means of a Bruker Smart Breeze CCD diffractometer operating with graphite-monochromated Mo- $K_{\alpha}$ radiation. A crystal of the product was glued at the end of a glass fibre. The intensities were corrected for Lorentz and polarisation effects and for absorption by means of a multi-scan method. ${ }^{1}$ Some relevant crystal parameters are listed in Table S1. The structure was readily solved by direct methods and subsequent difference Fourier techniques. The SHELXS-97 ${ }^{1,2}$ software package was used for solution, and SHELXL-97 ${ }^{2 \mathrm{~b}}$ was used for the refinement. All non-hydrogen atoms were refined anisotropically. Hydrogen atoms were placed at the calculated positions and refined as riding atoms with isotropic displacement parameters. Other control calculations and preparation of publication material were performed with the programs contained in the suite WINGX. ${ }^{3}$ Some final reliability factors are listed in Table S1.

Table S1. Crystal Data and Structure Refinement details for [Tb(dbm) $\left.)_{3}(\mathrm{DME})\right]$

$\begin{array}{ll}\text { formula } & \mathrm{C}_{49} \mathrm{H}_{43} \mathrm{O}_{8} \mathrm{~Tb} \\ F w & 918.75 \\ T(\mathrm{~K}) & 296 \\ \text { crystal system } & \text { monoclinic } \\ \text { space group } & P 2_{1} / c \\ a(\AA) & 12.4350(6) \\ b(\AA) & 23.7780(13) \\ c(\AA) & 14.7265(8) \\ \beta\left({ }^{\circ}\right) & 104.597(2) \\ V\left(\AA^{3}\right) & 4213.8(4) \\ Z & 4 \\ \text { reflections collected }\left(2 \theta_{\max }\right) & 29.99 \\ \rho(\text { calcd })\left(\mathrm{g} / \mathrm{cm}^{3}\right) & 1.448 \\ \text { unique refl. } & 12225 \\ \text { refl. }[I>2 \sigma(I)] & 9564 \\ \mu\left(\mathrm{mm}^{-1}\right) & 1.732 \\ \lambda(\AA) & 0.71073 \\ F(000) & 1864 \\ R 1^{\mathrm{a}} / \mathrm{GOF} & 0.0395 / 1.087 \\ w R 2^{\mathrm{c}}[I>2 \sigma(I)] & 0.0968 \\ \text { no. of param. } / \text { restraints } & 523 / 0 \\ \text { residual density }\left(e \cdot \AA^{3}\right) & 1.914 /-1.441 \\ { }^{\mathrm{a}} R 1=\Sigma\left\|F_{\mathrm{o}}|-| F_{\mathrm{c}}\right\| / \Sigma \mid F_{\mathrm{o}},{ }^{\mathrm{b}} \mathrm{GOF}=\left\{\Sigma\left[w\left(F_{\mathrm{o}}{ }^{2}-F_{\mathrm{c}}{ }^{2}\right)^{2}\right] /(N-P)\right\}^{1 / 2},{ }^{\mathrm{c}} w R 2 \\ =\left\{\Sigma\left[w\left(F_{\mathrm{o}}{ }^{2}-F_{\mathrm{c}}{ }^{2}\right)\right] / \Sigma\left[w\left(F_{\mathrm{o}}{ }^{2}\right)\right]\right\}^{1 / 2}, \text { where } w=1 /\left[\sigma^{2}\left(F_{\mathrm{o}}{ }^{2}\right)+(a Q)^{2}+\right. \\ b Q], Q=\left(\mathrm{MAX}\left(F_{\mathrm{o}}{ }^{2}, 0\right)+2 F_{\mathrm{c}}{ }^{2}\right) / 3\end{array}$


The molecular structure of $\left[\mathrm{Tb}(\mathrm{dbm})_{3}(\mathrm{DME})\right]$ is shown in Figure S1. The bond lengths and the more relevant bond angles around the metal are listed in Table S2. The coordination geometry may be described as distorted square antiprismatic, as it happens for the main part of octa-coordinated terbium complexes containing four bidentate oxygen ligands. ${ }^{4}$ The oxygen atoms of two dibenzoylmethanato ( $\mathrm{dbm}$ ) ligands define one square face of the antiprism and the other is defined by the oxygen atoms of the third dbm and of the dimethoxyethane unit. As it can be seen in Table $\mathrm{S} 2$, the $\mathrm{Tb}-\mathrm{O}$ distances from dbm ligands are in the range 2.31-2.36 $\AA$, while the $\mathrm{Tb}-\mathrm{O}$ distances from dimethoxyethane are significantly longer (2.53 and $2.57 \AA)$.

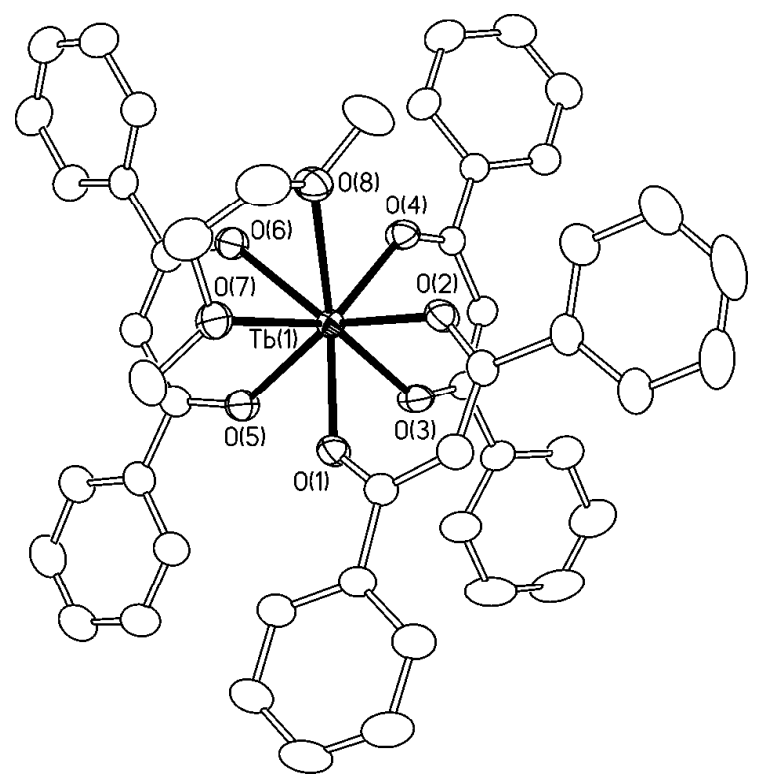

Figure S1. Molecular geometry of $\left[\mathrm{Tb}(\mathrm{dbm})_{3}(\mathrm{DME})\right]$ (30\% thermal ellipsoids; hydrogen atoms are omitted for clarity).

\begin{tabular}{|c|c|c|c|}
\hline $\mathrm{Tb}(1)-\mathrm{O}(1)$ & $2.322(2)$ & $\mathrm{Tb}(1)-\mathrm{O}(5)$ & $2.310(2)$ \\
\hline $\mathrm{Tb}(1)-\mathrm{O}(2)$ & $2.307(2)$ & $\mathrm{Tb}(1)-\mathrm{O}(6)$ & $2.357(2)$ \\
\hline $\mathrm{Tb}(1)-\mathrm{O}(3)$ & $2.320(2)$ & $\mathrm{Tb}(1)-\mathrm{O}(7)$ & $2.528(2)$ \\
\hline $\mathrm{Tb}(1)-\mathrm{O}(4)$ & $2.322(2)$ & $\mathrm{Tb}(1)-\mathrm{O}(8)$ & $2.570(2)$ \\
\hline $\mathrm{O}(1)-\mathrm{Tb}(1)-\mathrm{O}(2)$ & $73.00(7)$ & $\mathrm{O}(3)-\mathrm{Tb}(1)-\mathrm{O}(4)$ & $72.29(8)$ \\
\hline $\mathrm{O}(1)-\mathrm{Tb}(1)-\mathrm{O}(7)$ & $71.73(8)$ & $\mathrm{O}(3)-\mathrm{Tb}(1)-\mathrm{O}(5)$ & $77.65(8)$ \\
\hline $\mathrm{O}(2)-\mathrm{Tb}(1)-\mathrm{O}(8)$ & $72.78(8)$ & $\mathrm{O}(5)-\mathrm{Tb}(1)-\mathrm{O}(6)$ & $70.46(7)$ \\
\hline $\mathrm{O}(7)-\mathrm{Tb}(1)-\mathrm{O}(8)$ & $63.55(8)$ & $\mathrm{O}(4)-\mathrm{Tb}(1)-\mathrm{O}(6)$ & $75.20(8)$ \\
\hline $\mathrm{O}(1)-\mathrm{Tb}(1)-\mathrm{O}(3)$ & $78.65(8)$ & $\mathrm{O}(8)-\mathrm{Tb}(1)-\mathrm{O}(6)$ & 71.01(8) \\
\hline $\mathrm{O}(2)-\mathrm{Tb}(1)-\mathrm{O}(3)$ & $86.50(8)$ & $\mathrm{O}(6)-\mathrm{Tb}(1)-\mathrm{O}(7)$ & $78.85(8)$ \\
\hline $\mathrm{O}(2)-\mathrm{Tb}(1)-\mathrm{O}(4)$ & $86.93(8)$ & $\mathrm{O}(5)-\mathrm{Tb}(1)-\mathrm{O}(7)$ & $84.43(8)$ \\
\hline $\mathrm{O}(4)-\mathrm{Tb}(1)-\mathrm{O}(8)$ & $78.46(8)$ & $\mathrm{O}(1)-\mathrm{Tb}(1)-\mathrm{O}(5)$ & $75.40(7)$ \\
\hline
\end{tabular}




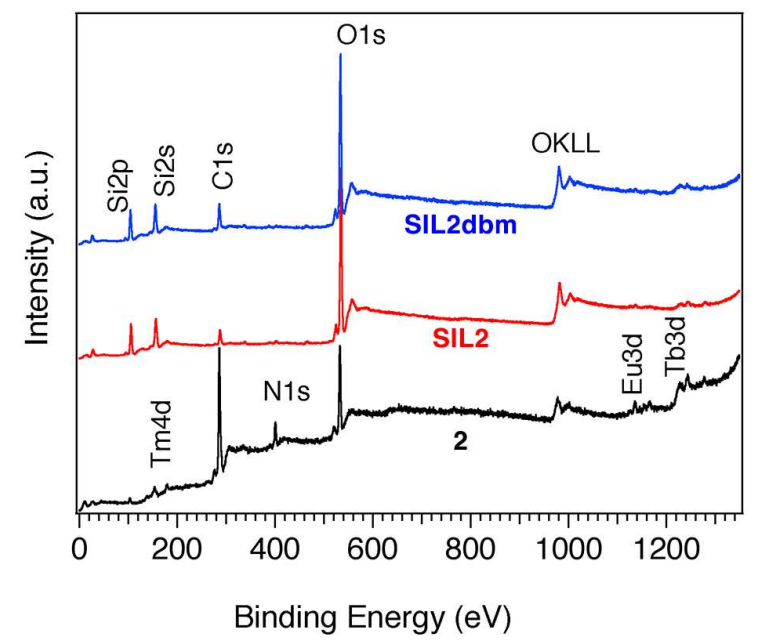

Figure S2. Survey spectra of 2, SIL2 and SIL2dbm. The principal bands are labeled in the figure.

\section{Reference list}

${ }^{1}$ Sheldrick, G. M. SADABS, Program for empirical absorption correction, University of Göttingen, Germany, 1996.

2 (a) Sheldrick, G. M. ShelXS97, Programs for Crystal Structure Solution; University of Göttingen, Göttingen, Germany, 1997. (b) Sheldrick, G. M. ShelXL97, Programs for Crystal Structure Refinement; University of Göttingen, Götingen, Germany, 1997.

${ }^{3}$ Farrugia, L. J. J. Appl. Crystallogr., 1999, 32, 837-838.

${ }^{4}$ (a) Drake, S. R.; Lyons, A.; Otway, D. J.; Slawin, A. M. Z.; Williams, D. J. J. Chem. Soc., Dalton Trans., 1993, 23792386. (b) Eliseeva, S. V.; Ryazanov, M.; Gumy, F.; Troyanov, S. I.; Lepnev, L. S.; Bünzli, J.-C. G.; Kuzmina, N. P. Eur. J. Inorg. Chem. 2006, 4809-4820. (c) Eliseeva, S. V.; Kotova, O. V.; Gumy, F.; Semenov, S. N.; Kessler, V. G.; Lepnev, L. S.; Bünzli, J.-C. G.; Kuzmina, N. P. J. Phys. Chem. A 2008, 112, 3614-3626. (d) Tang, Si-Fu; Mudring, A.V. Eur. J. Inorg. Chem. 2009, 2769-2775. 